\title{
Application of laser remote sensing technology and super continuous spectrum laser
}

\author{
Li Jing ${ }^{1,2 *}$, Che Ying ${ }^{1}$, Jin Meishan², Zhai Yannan², Ding Changhong ${ }^{2}$ \\ ${ }^{1}$ School of Photoelectric Engineering, Changchun University of Science and Technology,Changchun, Jilin 130022, China \\ ${ }^{2}$ Aviation University of Air Force, Changchun, Jilin 130022, China
}

\begin{abstract}
Fiber optic super continuous spectrum laser technology is a new technology developed in recent years. It takes into account the advantages of good alignment of laser source, high energy density and wide wavelength range of ordinary white light source, which can not only accurately remote sense distant target, but also obtain hyperspectral information of distant target. Super continuous spectrum of laser radar is a kind of remote sensing monitoring instrument, it can obtain three-dimensional spectral information of the target, and can be accurately detected in the night of distant high spectral information, the all-weather, three-dimensional spectrum detection method with the traditional passive remote sensing compared with single/multiband laser radar technology has incomparable advantages. Based on the development trend of lidar abroad, the development status of super-continuous spectrum lidar in China and the problems in remote sensing detection of oil spill, this paper introduces the feasibility of using super-continuous spectrum laser to monitor oil spill in the sea, which lays a foundation for further research.
\end{abstract}

\section{Introduction}

Remote sensing technology is a detection technology developed in the 1960s. It is a technology that uses passive or active means to obtain the electromagnetic waves reflected, scattered or radiated by the distant target, so as to detect and identify the target without contact ${ }^{[1]}$. Remote sensing technology has been widely used in the atmosphere, ocean, resources, agriculture, forestry, military and other aspects. The development and progress of remote sensing technology is closely related to people's lives. With the development of laser technology, especially the appearance of tunable laser and super continuous spectrum laser, lidar is not only limited to the field of atmospheric remote sensing, but also presents unique advantages in ocean exploration, wind field remote sensing, ground object recognition and detection. Due to its high energy density, good directivity and wide measurement spectrum, super continuous spectrum lidar has unique advantages over traditional single-band and multi-band lidar, so it has gradually become a new research focus.

\section{Development of lidar abroad}

Since the first ruby laser was developed in 1960s, it has attracted people's attention because of its different characteristics from the traditional light source and has been widely used in various fields. Laser is widely used in various fields because of its characteristics different from traditional light sources, including good monochromatism and directivity, and high brightness.
Compared with traditional electromagnetic wave detection methods, laser has a much shorter wavelength, which can interact with all kinds of tiny particles in the atmosphere and detect the medium distribution on the laser transmission path, it has a good direction and a high energy density, which can ensure its transmission distance in the atmosphere, so it has advantages that cannot be surpassed by traditional atmospheric detection methods. Since the birth of laser, some scholars have proposed that it can be combined with traditional radar technology and applied in the field of atmospheric remote sensing ${ }^{[2]}$.

After 50 years of development, with the progress of laser technology and the development of the theory of detecting, laser radar technology has been widely applied in the atmospheric wind field of remote sensing, ocean exploration, target detection and object detection, and other fields, in the city of fog monitoring, urban biochemical counterterrorism, weather forecast, the respect such as precision agriculture reflects the absolute advantage. According to the different principles and development stages of lidar detection, it can be divided into three types: single-wavelength detection, multiwavelength detection and super-continuous spectrum detection.

\subsection{Single wavelength detection}

Single-wavelength detection refers to the laser source has only a fixed pumping wavelength, through the laser and the atmosphere and the ocean particles, gas molecules, observe the characteristics of the echo

\footnotetext{
* Corresponding author: 568026388@qq.com
} 
changes to achieve the remote sensing of the detection target. According to the different principle, the singlewavelength detection lidar can be divided into meter scattering, Rayleigh scattering, depolarization scattering, Raman scattering, hyperspectral resolution, resonance fluorescence and incoherent doppler frequency shift lidar.

In 1963, Fiocco and Smullin used ruby laser to make lidar observations of the atmospheric mesosphere and reported the spatial distribution of atmospheric aerosols in the troposphere ${ }^{[3]}$. In the upper atmosphere, the content of aerosol is very small, so Rayleigh scattering accounts for a larger proportion in the echo of the upper atmosphere, so Rayleigh scattering can be used to invert the temperature of the upper atmosphere.

In 2001, John w. Hair of NASA, successfully separated the meter scattering signal from the Rayleigh scattering signal by iodine vapor filter ${ }^{[4]}$. The structure of the receiving system of this high-resolution lidar is shown in Fig. 1. The signals received by the telescope are fed into the detection box through a multimode optical fiber.The signal beam is divided into two channels, one channel has iodine vapor filter, the other channel has no filter, so as to realize the distinction between the meter scattering signal and Rayleigh scattering signal.

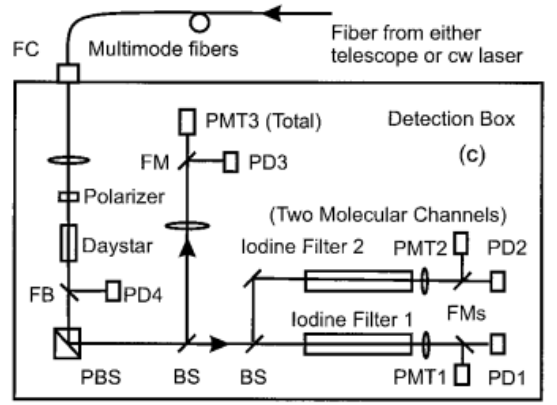

Fig. 1. receiver system structure of high-resolution radar.

Raman scattering lidar technology uses the vibration and rotational energy levels of molecules to measure the extinction coefficient of aerosols , temperature and concentration of gas molecules such as water vapor and can also be used to detect the temperature of seawater. A typical Raman lidar using Nd: YAG laser as the light source is shown in Fig. 2, which was developed by Whiteman et al. In $1992^{[5]}$. The laser radar transmitter and the receiving optical system are independent, in which the light source is a pulse laser with an outgoing wavelength of $354.7 \mathrm{~nm}$ and an energy of $150 \mathrm{~mJ}$. The receiving device adopts a 0.75 meter aperture, f/4.8 cassegreen telescope, and its observation Angle can be adjusted within the range of $0.3 \sim 5 \mathrm{mrad}$.

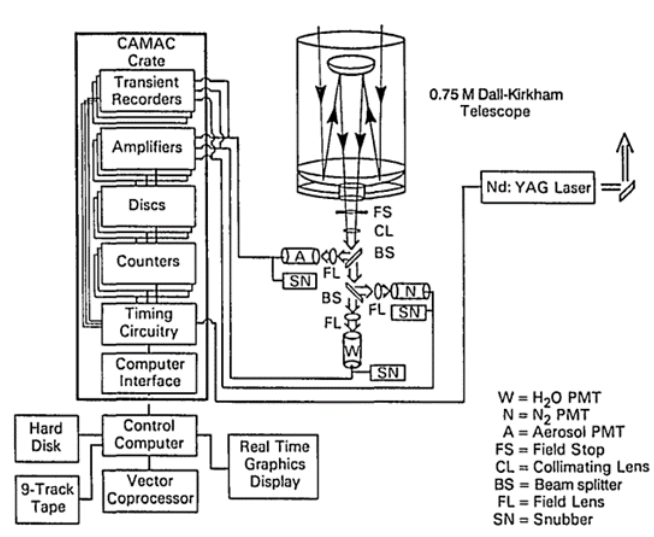

Fig. 2. Raman lidar optical schematic.

From the above analysis, it can be seen that the single-wavelength detection technology has gradually developed from meter scattering and Rayleigh scattering lidar technology, which can only be used for atmospheric aerosol detection and upper atmospheric temperature detection, to a variety of lidar technologies that can detect atmospheric gas composition, nearformation atmospheric temperature and wind field. For Raman scattering radar and hyperspectral resolution radar, in order to improve the measurement accuracy, the bandwidth of the output laser needs to be as narrow as possible ${ }^{[6]}$, and single-wavelength detection is gradually unable to meet the detection requirements.

\subsection{Multiwavelength detection}

With the development of lidar technology, especially the appearance of tunable laser, not only the previous lidar technology has been improved, but also many new lidar detection technologies, such as fluorescence lidar technology and differential absorption lidar technology. There are two kinds of fluorescent lidar technologies, including resonance fluorescence and excitation fluorescence. Resonance fluorescence lidar technology is mainly applied to ion layer detection in the middle and upper atmosphere, and $\mathrm{Na}$ atom is a good tracer to characterize the fluctuation in the middle and upper atmosphere ${ }^{[7]}$. At present, resonance fluorescence radar mainly carries out detection in the upper $\mathrm{Na}$ layer. Thomas and Paul et al. from Canada designed a resonant fluorescent lidar using a tunable laser, and its transmitter is shown in Fig. 3. Its light source uses a tunable laser pumped by $532 \mathrm{~nm} \mathrm{Nd}$ : YAG laser, which can be tuned in the $330-710 \mathrm{~nm}$ band. The $589 \mathrm{~nm}$ band is selected as the working wavelength. The resulting laser has a pulse width of $10 \mathrm{~ns}$ and a capacity of about $100 \mathrm{~mJ}$.Using a concave lens and launching telescope, the laser beam is amplified to a diameter of $12 \mathrm{~cm}$, with a beam spread ratio of about 30 times. By changing the distance between its concave lens and the objective lens, the beam can be shifted from a diffraction limited parallel beam to a maximum of $0.7 \mathrm{arc}$ minutes. The receiver is a schmidt - cassegrain telescope with a diameter of $15 \mathrm{~cm}$ and the detector is a CCD. 


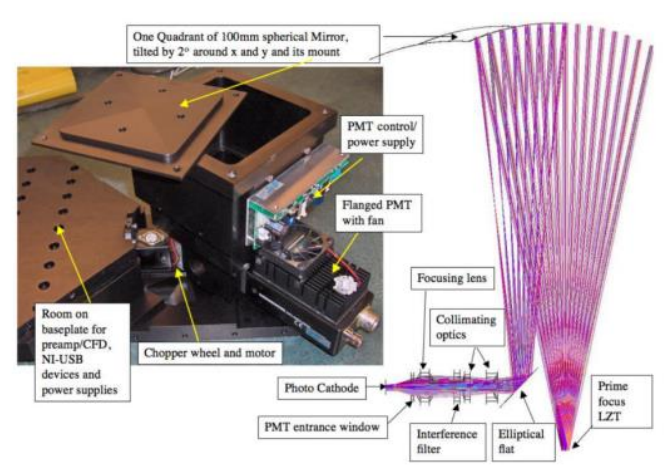

Fig. 3. Resonance fluorescent lidar.

Traditional lidar is limited by the single laser spectrum. Different types of pump sources determine the output of laser wavelength. However, tunable lasers are very expensive and expensive to use, and although tunable lasers have a large tuning range, only one wavelength can be emitted at a timeWith the emergence of femtosecond laser excitation super-continuous spectrum and optical fiber super-continuous spectrum laser light source, multi-line difference technology has become possible' and it has unique advantages compared with traditional spectrometer remote sensing technology in ground object recognition, so super-continuous spectrum lidar has gradually become a new research hotspot.

\subsection{Supercontinuous spectrum detection}

There are mainly two types of super-continuous spectrum lidar. One is to generate continuous laser pulses with wide spectrum by utilizing cascade nonlinear optical fiber interaction in the light, including four-wave mixing and nonlinear optics, and is called hyperspectral scanning lidar; The other is the use of femtosecond laser emitted by the strong laser and atmospheric media have a strong nonlinear effect, the generation of a wide spectrum of continuous laser pulse, known as broadband coherent white light continuum differential absorption laser radar (DIAL). Super continuous spectrum laser has the characteristics of high energy and good direction as well as wide spectrum of white light source, so it is also called white light laser. At present, there are three main application fields of super-continuous spectrum lidar, which are atmospheric sounding, tracking target jamming and ground object target recognition.

T. Somekawa of Japan institute of laser technology and n. Manago et al. of Japan center for environmental remote sensing used DIAL to detect the distribution profile of $\mathrm{H} 2 \mathrm{O}$ and $\mathrm{O} 2$ in troposphere in 2016 .DIAL experimental Settings are shown in Fig. 4 (a). The experiment adopted the CPA laser system with an output energy of about $100 \mathrm{mj} /$ pulse, a pulse width of $100 \mathrm{fs}$ and a repetition frequency of $10 \mathrm{hz}$. Instead of using the traditional method of femtosecond laser interacting with air to produce a supercontinuum, they focused the femtosecond laser through a five-meter focal length lens into a 9-meter krypton (Kr) gas chamber. The supercontinuous spectrum generated by femtosecond laser interaction with air and krypton gas is shown in Fig.
4 (b). It can be seen that krypton gas generates stronger supercontinuous spectrum energy in visible bands than air.

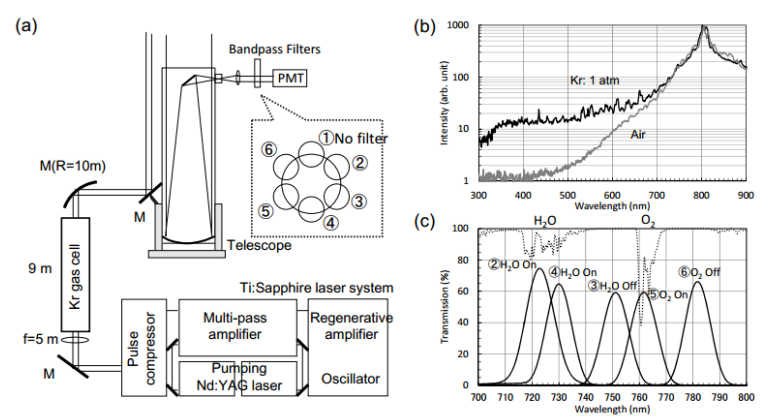

Fig. 4. Experimental setup and analysis of experimental results.

The device is used to measure the water vapor and $\mathrm{O} 2$ in the near-infrared band. The receiving device is shown in Fig. 4 (a). It adopts the structure of Newton telescope and has a diameter of $30 \mathrm{~cm}$. The detection wavelength is selected by rotating the filter wheel, and the detection results are shown in Fig. 4 (c). As lidar, it may be able to be used for the detection and recognition of weak reflectivity targets such as minerals. Ground object target detection is mainly aimed at strong reflectivity targets, such as vegetation detection and oil spill monitoring on the sea surface. Some satellite-based lidar systems can also be used for atmospheric and land surface research. Teemu and Juha et al. of the institute of geosmology of Finland first proposed the concept of hyperspectral scanning lidar in 201 years ${ }^{[8]}$. They combine a super-continuous spectrum laser scanner with a hyperspectral imager to achieve three-dimensional spectral scanning of vegetation. The structural design of the hyperspectral scanning lidar is shown in Fig. 5:

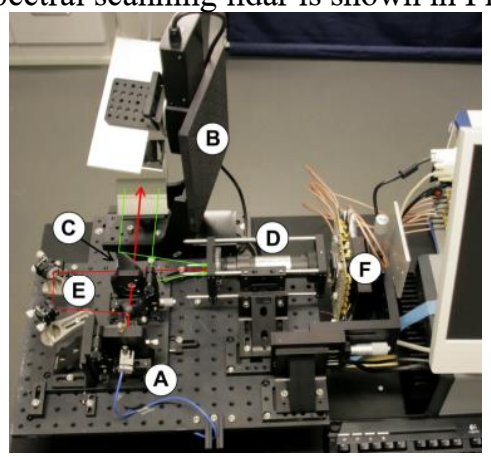

Fig. 5. Structural design of hyperspectral scanning lidar.

The ultra-continuous laser adopted by the instrument has a pulse width of $1 \mathrm{~ns}$, a repetition frequency of $24 \mathrm{khz}$, and an average power of $100 \mathrm{mw}$ in the spectral range of 480-2200nm. The spectral range is shown in Fig. 6. After the laser beam emitted by the photonic crystal fiber (A) is split, part of the laser beam directly passes through the hole in the off-axis parabolic mirror $(\mathrm{C})$, and then passes through the two-dimensional scanner (B) to scan and detect the ground object target after colocation. The other part of the light passes through the wavelength splitter (E) and enters the spectrometer directly through a hole in the off-axis parabolic mirror. 


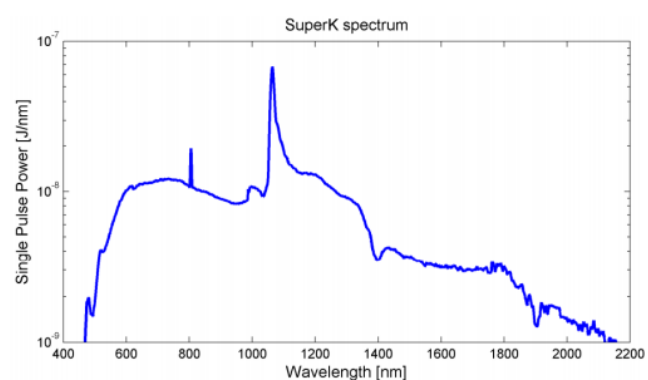

Fig. 6. Hyperspectral scanning lidar spectral range.

The spectrometer detector used by the instrument has a temporal resolution of about $4 \mathrm{~ns}$, that is, a vertical resolution of about $0.6 \mathrm{~m}$ in the direction of laser emission. This lidar was used to detect the spruce in the dark environment and generate a three dimensional spectral point cloud image. The measurement results obtained by lidar are compared with the results of measuring spruce canopy and trunk under the same conditions with the spectrograph illuminated by halogen tungsten lamp. The comparison results are shown in Fig. 7. The detection results have a good consistency.

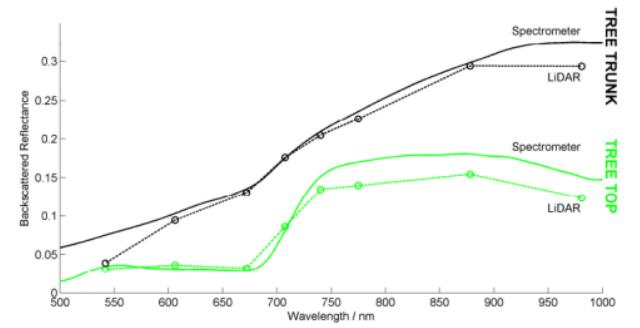

Fig. 7. Schematic diagram of comparison results.

Wide-band coherent white continuous medium differential absorption lidar technology can produce extremely high energy laser pulses, so it has attracted the attention of scholars in the field of atmospheric sounding, but its light source is expensive and large, and its application scope is limited. Based on fiber optic super continuous spectrum laser, hyperspectral scanning lidar can excite wide-spectrum laser in uv-visible infrared band, which is cheap and small in size, and has a good development prospect in airborne lidar detection field and even satellite-borne laser detection field. However, the outstanding problem of fiber super-continuous spectrum laser lies in its low pulse energy, and in order to obtain a complete echo waveform, the repetition frequency and pulse width of the super-continuous spectrum laser need to be limited ${ }^{[9]}$. Therefore, it is necessary to improve the detection range of the fiber supercontinuum laser.

\section{Current situation and development trend of lidar in China}

In 1966, the institute of atmospheric sciences of Chinese academy of sciences and Shanghai institute of optical machinery jointly developed the first meter-scattering ruby lidar in China.However, it was not until the 1990s that various laser radars were gradually developed and applied .
Since 2000, with the support of the national 863 project, zhou jun et al. of anhui institute of optics and fine mechanics, Chinese academy of sciences, have developed a series of vehicle-mounted meter-scattering lidar systems . Nd: YAG laser is used as the light source to detect atmospheric aerosols at $1064 \mathrm{~nm}$ and 532nm. Yang junjun et al. of anhui institute of optics and fine mechanics, Chinese academy of sciences, successfully developed China's first vehicle-mounted laser pollution detection radar in 2004, which uses a tunable laser as the light source and uses differential absorption technology to identify pollutant gases and aerosols and detect their concentrations . In 2006, liu zhishen et al. from ocean university of China successfully developed China's first vehicle-borne incoherent doppler lidar for wind speed measurement, and improved the accuracy of wind speed inversion by improving wind field measurement and inversion method ${ }^{[10]}$, which was tested in the Qingdao international sailing race in 2007.

As an emerging technology, super continuous spectrum lidar (SRS) has a late start in China, and there are few related researches. In 2008, zhang liwen et al. of wuhan institute of physics and mathematics, Chinese academy of sciences, built China's first white light lidar with DIAL technology, and the detection results were consistent with the fitting value of Hitran database in the United States. In 2016, li feng et al. of the school of optics and electronics of the Chinese academy of sciences built a hyperspectral scanning lidar based on microlens array, which solved the problem of limited spectral channel of super-continuous spectrum lidar [11]. In 2017, feng mingbo and others from the institute of remote sensing and digital earth of the Chinese academy of sciences built a hyperspectral scanning lidar with a resolution of $0.2 \mathrm{~m}$ in the direction of emission, and analyzed the observation results of vegetation targets with strong reflectivity in a darkroom environment. The analysis results show that the vegetation $3 \mathrm{~d}$ spectrum obtained by super-continuous spectrum lidar can reflect the physiological and biochemical characteristics of vegetation $3 \mathrm{~d}$ levels .

As a continuous spectrum of laser radar, high spectral scanning laser radar has both traditional laser radar high energy and good direction, can realize the advantages of distance detection, also has the advantage of the white light source spectral spectrum width, so in the threedimensional three-dimensional spectral reflectance imaging and weak target recognition has a good application prospect. At present, hyperspectral scanning lidar still has such problems as low pulse energy, being susceptible to interference from ambient light and limited spectral channels, which can only be used for laboratory darkroom measurement.We should actively seize the opportunity to seize the scientific high ground.

\section{Current situation of remote sensing monitoring of oil spill on sea surface}

China's economy has developed rapidly in recent years and has made many remarkable achievements. In this development process, the demand for crude oil is 
increasing day by day, and sea transportation is an important way of oil transportation, because of oil pipeline rupture, tanker accident caused by oil spill accidents occur from time to time. In 2018, the changfeng crystal collided with the Panamanian tanker sanchi about 160 nautical miles east of the Yangtze estuary. The sanchi, carrying 136,000 tons of condensate and 1,000 tons of heavy diesel, burned and eventually exploded. The spilt material spread to the sea of Japan along with the tsushima current, causing great harm to the ecological environment and the safety of people's property. The occurrence of oil spill on the sea surface will cause great harm to the Marine natural ecology and Marine living resources, and even affect Marine industries such as mariculture, fishery and tourism, which will seriously threaten people's life and property ${ }^{[12]}$. Therefore, it is of great significance to carry out timely and accurate monitoring of the occurrence of Marine oil spill.

Since the oil spill is an emergency, the time and place of its occurrence are random, so it is necessary to monitor its occurrence 24 hours a day. Currently, the commonly used methods for monitoring oil spill on the sea surface are mainly remote sensing methods, and no method is fully competent for monitoring oil spill on the sea surface at present ${ }^{[13]}$. The synthetic aperture radar (SAR) using microwave radiation remote sensing technology has the advantages of large detection range and 24-hour continuous operation. However, its resolution is low and the oil film thickness cannot be accurately determined. Hyperspectral technology has the highest resolution and can distinguish true and false targets. Near-infrared remote sensing technology has the advantages of high reliability and fast speed, but it is difficult to distinguish the thickness of oil film, and the ultraviolet band also has a high radiation degree for the extremely thin oil layer, but the ultraviolet band is rapidly attenuating in the atmosphere and cannot be used for remote sensing. By emitting ultraviolet light of a specific band, laser fluorescence method can stimulate oil spill to generate fluorescence, so as to realize roundthe-clock continuous detection of oil spill. However, the fluorescence is weak and susceptible to interference, so its accuracy is not high. Currently, supercontinuous spectrum laser has not been applied to the monitoring of oil spill at sea, as a new technology developed in recent years, super continuous spectrum lidar has many problems, such as low pulse energy, easy to be interfered by ambient light and limited spectral channel.

\section{References}

1. Jensen $\mathrm{J}$ R, Lulla K. Introductory digital image processing: a remote sensing perspective[J]. 1987.

2. HONEY R C, EVANS W E. Laser radar (lidar) for meteorological observations, Review of Scientific Instruments[J], 1966. 37(4): 393-400
3. Fiocco G, Smullin L D. Detection of scattering layers in the upper atmosphere $(60-140 \mathrm{~km})$ by optical radar[J]. Nature, 1963, 199(4900): 1275.

4. Hair J W, Caldwell L M, Krueger D A, et al. Highspectral-resolution lidar with iodine-vapor filters: measurement of atmospheric-state and aerosol profiles[J]. Applied optics, 2001, 40(30): 5280-5294.

5. Whiteman, D. N. et al. "Raman lidar system for the measurement of water vapor and aerosols in the Earth's atmosphere.” Applied optics 3116 (1992): 3068-82.

6. Müller D, Ansmann A, Mattis I, et al. Aerosol-type - dependent lidar ratios observed with Raman lidar[J]. Journal of Geophysical Research: Atmospheres, 2007, 112(D16).

7. Bowman M R, Gibson A J, Sandford M C W. Observation of mesospheric $\mathrm{Na}$ atoms by tuner laser radar[J]. Nature, 1969, 221(1): 456-458.

8. Hakala T, Suomalainen J, Kaasalainen S, et al. Full waveform hyperspectral LiDAR for terrestrial laser scanning[J]. Optics express, 2012, 20(7): 7119-7127.

9. Zhen Wang, Chuanrong Li, Mei Zhou, Huijing Zhang, Wenjing He, Wei Li, Yuanyuan Qiu, "Recent development of hyperspectral LiDAR using supercontinuum laser ," Proc. SPIE 10156, Hyperspectral Remote Sensing Applications and Environmental Monitoring and Safety Testing Technology, 101560I (25 October 2016)

10. Liu Z S, Liu B Y, Li Z G, et al. Wind measurements with incoherent Doppler lidar based on iodine filters at night and day[J]. Applied Physics B, 2007, 88(2): 327-335.

11. Feng Li, Chenghao Jiang, Jingguo Zhu, Menglin Li, Zhe Meng, "Research on high resolution spectral method of hyperspectral LiDAR," Proc. SPIE 10156, Hyperspectral Remote Sensing Applications and Environmental Monitoring and Safety Testing Technology, 101561X (25 October 2016)

12. Alam M S, Sidike P. Trends in oil spill detection via hyperspectral imaging[C]//2012 7th International Conference on Electrical and Computer Engineering. IEEE, 2012: 858-862.

13. Lang $\mathrm{H}$, Zhang $\mathrm{X}, \mathrm{Xi} \mathrm{Y}$, et al. Dark-spot segmentation for oil spill detection based on multifeature fusion classification in single-pol synthetic aperture radar imagery[J]. Journal of Applied Remote Sensing, 2017, 11(1): 015006. 